\title{
Progressive thermopreconditioning attenuates rat cardiac ischemia/reperfusion injury by mitochondria-mediated antioxidant and antiapoptotic mechanisms
}

\author{
Chen-Yen Chien, MD, ${ }^{\mathrm{a}, \mathrm{b}, \mathrm{c}}$ Chiang-Ting Chien, $\mathrm{PhD},{ }^{\mathrm{d}}$ and Shoei-Shen Wang, $\mathrm{MD}^{\mathrm{e}}$
}

Objectives: Progressive thermal preconditioning (PTP) provides vascular protection with less hemodynamic fluctuations, endoplasmic reticulum (ER), and oxidative stress compared with whole body hyperthermia. We suggest PTP might efficiently diminish cardiac ischemia/reperfusion-induced apoptosis and autophagy injury.

Methods: A total of 67 male Wistar rats were divided into a non-PTP control group, 24 or 72 hours after a single cycle or 3 consecutive cycles of PTP in a $42^{\circ} \mathrm{C}$ water bath (1-24, 1-72, 3-24, and 3-72 groups). We measured the cardiac $\mathrm{O}_{2}{ }^{-}$amount in vivo in response to left anterior descending coronary artery ligation for 2 hours and reperfusion for 3 hours. Cardiac function and injury were determined by microcirculation, electrocardiography, and infarct size. The PTP-induced protective effects on nicotinamide adenine dinucleotide phosphate oxidase gp91-mediated oxidative stress, ER stress, and apoptosis- and autophagy-related mechanisms were examined using Western blot and immunohistochemistry.

Results: Coronary arterial ischemia/reperfusion depressed cardiac microcirculation, induced ST-segment elevation and increased infarct size in non-PTP and PTP rats. Ischemia/reperfusion enhanced the cardiac $\mathrm{O}_{2}{ }^{-}$levels by enhanced nicotinamide adenine dinucleotide phosphate oxidase gp91 expression, cytosolic cytochrome $\mathrm{C}$ release, and decreased mitochondrial Bcl-2 expression. Cardiac injury activated ER stress-78-kDa glucose-regulated protein expression, increased the $\mathrm{Bax} / \mathrm{Bcl}-2$ ratio, cleaved caspase 3 expression and poly(ADP-ribose)-polymerase fragments, leading to apoptosis formation, and promoted LC3-II expression, resulting in autophagy formation. PTP treatment elevated heat shock protein 70 , heat shock protein $32, \mathrm{Bcl}-2, \mathrm{Bcl}-\mathrm{xL}$, and manganese superoxide dismutase in the rat heart, especially in the 3-72 group. PTP treatment significantly restored cardiac microcirculation, decreased oxidative stress, ER stress, apoptosis, autophagy, and infarct size.

Conclusions: PTP significantly reduced cardiac ischemia/reperfusion injury by upregulating antioxidant, antiapoptotic, and antiautophagic mechanisms. (J Thorac Cardiovasc Surg 2014;148:705-13)

The burst production of reactive oxygen species (ROS) during IR stage might impair the function and structure of the tissue or organ by triggering several abnormal signal

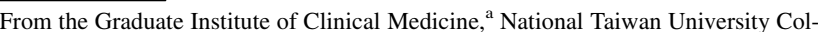
lege of Medicine, Taipei, Taiwan, Republic of China; Department of Surgery, ${ }^{b}$ Mackay Memorial Hospital and Mackay Medical College, Taipei, Taiwan, Republic of China; Mackay Medicine, ${ }^{\mathrm{c}}$ Nursing and Management College, New Taipei City, Taiwan, Republic of China; Department of Biological Science, ${ }^{\mathrm{d}}$ National Taiwan Normal University, Taipei, Taiwan, Republic of China; Department of Surgery, ${ }^{\mathrm{e}}$ National Taiwan University Hospital and College of Medicine, Taipei, Taiwan, Republic of China.

This work was supported in part by the National Science Council of the Republic of China (NSC 102-2320-B-003-001-MY3).

Disclosures: Authors have nothing to disclose with regard to commercial support.

Dr C.-T. Chien and Dr Wang contributed equally to this work.

Dr C.-Y. Chien is currently at the Department of Surgery, Mackay Memorial Hospital and Mackay Medical College, Taipei City, Taiwan, Republic of China; Dr C.-T. Chien is currently at Department of Life Science, National Taiwan Normal University, Taipei City, Taiwan, Republic of China.

Received for publication Sept 28, 2013; revisions received Nov 12, 2013; accepted for publication Dec 5, 2013; available ahead of print Feb 7, 2014.

Address for reprints: Chiang-Ting Chien, $\mathrm{PhD}$, Department of Life Science, National Taiwan Normal University, No 88, Tingzhou Rd, Taipei City, Taiwan 11677, Republic of China (E-mail: ctchien@ntnuh.edu.tw)

$0022-5223 / \$ 36.00$

Copyright (C) 2014 by The American Association for Thoracic Surgery

http://dx.doi.org/10.1016/j.jtcvs.2013.12.065
}

transductions to induce several types of cell death such as apoptosis, autophagy, pyroptosis, and necrosis. ${ }^{1-5}$ The increased ROS production enhanced Bax/Bcl-2/caspase 3/poly-(ADP-ribose)-polymerase signaling to trigger apoptosis, Beclin-1/LC3-II signaling to induce autophagy in ischemic and hypoxic tissues and cells. $^{1-5}$ Nicotinamide adenine dinucleotide phosphate (NADPH) oxidase and the mitochondrion contribute to the production of ROS in stressed cells. ${ }^{1-6}$ NADPH oxidase activation or NADPH oxidase subunit gp91 upregulation increases $\mathrm{O}_{2}{ }^{-}$generation in the rat heart. ${ }^{6,7}$ Mitochondrial dysfunction triggers cytosolic cytochrome $\mathrm{C}$ release, enhances excess $\mathrm{O}_{2}{ }^{-}$production, and promotes myocardial injury. ${ }^{8}$ Thus, decreased ROS production by inhibiting NADPH oxidase gp91 activity or restoring mitochondrial function might attenuate cardiac IR injury.

Enhancement of the endogenous defense mechanisms protects the cardiovascular system against ROS-induced injury. ${ }^{9}$ Thermal preconditioning is the most accessible method for inducing heat shock protein (HSP)-mediated resistance and tolerance to oxidative injury ${ }^{10,11}$ and confers cardiac protection. ${ }^{12}$ However, whole body hyperthermia by acute immersion of $\sim 80 \%$ of the total body surface into a hot water 


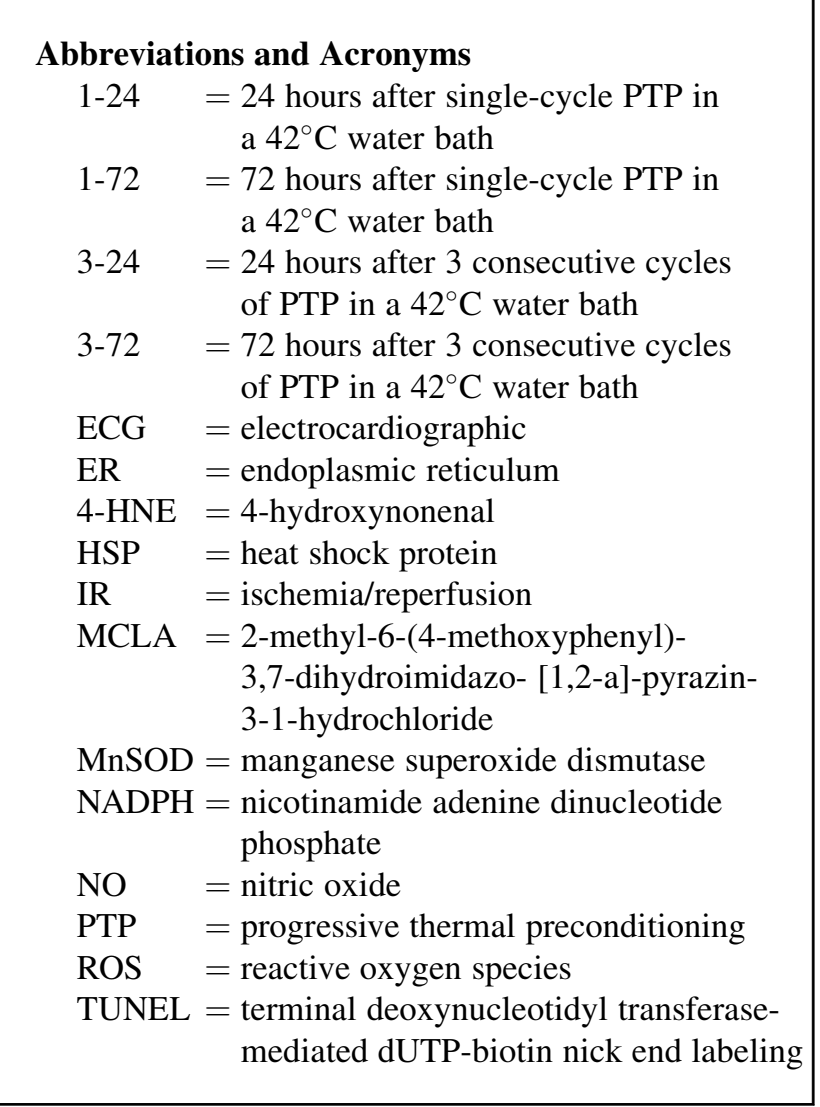

bath sometimes induces adverse effects, including acute myocardial and cerebral infarction by transient changes in blood pressure, heart rate, blood viscosity, fibrinolytic activity, and platelet function. ${ }^{13}$ It is possible that exposure to lesser heat challenges for a shorter period or gradually increasing the percentage of total body surface to heat stimulation would have similar and safe cardioprotective effects; however, this has not yet been determined. In humans and animals, repeated low-temperature sauna or thermal therapy can improve myocardial perfusion and vascular endothelial dysfunction. ${ }^{14,15}$ A modified method of progressive thermal preconditioning (PTP) with gradual increases in the body surface area by 3 steps of $42^{\circ} \mathrm{C}$ bathing can attenuate these adverse effects and confer efficiently vascular protection by way of phosphatidylinositol 3-kinase/Akt/endothelial nitric oxide synthase signaling pathways. ${ }^{16,17}$ Increased PTP frequency also enforces the vascular protection by the decreasing oxidative and endoplasmic reticulum (ER) stress, autophagy, apoptosis, and leukocyte infiltration. ${ }^{17}$ In contrast, regulation of antiapoptotic Bcl-2 and Bcl-xL expression or antioxidant manganese superoxide dismutase (MnSOD) could also be involved in PTP-induced protection. Bcl-2 family members such as Bax and Bcl-2 can regulate apoptosis and autophagy $\mathrm{y}^{3,18}$ by translocation of Bax or Bcl-2 to the mitochondria and regulate cytosolic cytochrome $\mathrm{C}$ release. ${ }^{19,20}$ MnSOD, mainly expressed in the mitochondria, can participate in the regulation of mitochondrial function and affect cardiac cell apoptosis and autophagy. However, limited data on PTP-induced antioxidant, antiapoptotic, and antiautophagic protection have been published. In the present study, we explored whether PTP provides cardiac protection against coronary IR injury by way of antioxidant, antiapoptotic, and antiautophagic action.

\section{METHODS}

\section{Animals}

A total of 67 male Wistar rats (weight, 220-240 g) were purchased from BioLASCO Taiwan Co, Ltd (Taipei, Taiwan) and housed in the Experimental Animal Center of National Taiwan Normal University. Food and water were provided ad libitum. The Institutional Animal Care and Use Committee of the National Taiwan Normal University had approved all the surgical and experimental procedures, which were in accordance with the guidelines of the National Science Council of the Republic of China (NSC 1997). All efforts were made to minimize animal suffering.

\section{Setup for PTP}

The models for PTP have been clearly described previously. ${ }^{16}$ In brief, the rats were immersed in tap water and loosely fixed to plastic lattice plates using adhesive tape, and the plates were set in a head-up position of $30^{\circ}$ to horizontal in a Plexiglas animal cage that was used as a bathtub. The temperature of the bathtub water was maintained at $42^{\circ} \mathrm{C}$ throughout the experiment by immersing the bathtub in a water bath incubator. The rats were then progressively immersed in the $42^{\circ} \mathrm{C}$ water bath at line $1(\sim 15 \%$ of the total body surface area) for 5 minutes, line 2 ( $\sim 40 \%$ of the total body surface area) for 5 minutes, and line 3 ( $\sim 70 \%$ of the total body surface area) for 5 minutes. Three consecutive cycles of PTP were performed within 8 hours and the interval between 2 PTP sessions was 4 hours. We divided these rats into 5 groups: (1) non-PTP control (exposed to air), (2) 1-24, 24 hours after a single cycle of PTP; (3) 1-72, 72 hours after a single cycle of PTP; (4) 3-24, 24 hours after 3 consecutive cycles of PTP; and (5) 3-72, 72 hours after 3 consecutive cycles of PTP. In response to cardiac IR injury, 2 rats in the non-PTP control, 2 in the 1-24, 2 in the 1-72, and 1 in the 3-24 group died. The final number in each group was 12 rats. The mortality rate was $10 \%$ (7 of 67 ).

\section{Measurement of Hemodynamic and Electrocardiographic Parameters}

Under urethane $(1.2 \mathrm{~g} / \mathrm{kg}$, intraperitoneally) anesthesia, the rat's trachea was intubated for artificial ventilation (Small Animal Ventilator Model 683, Harvard Apparatus, Holliston, Mass), with 50 breaths in 1 minute, a tidal volume of $8 \mathrm{~mL} / \mathrm{kg}$, and a positive end-expiratory pressure of $5 \mathrm{~cm}$ $\mathrm{H}_{2} \mathrm{O}$. The arterial blood pressure and heart rate measurements were recorded with a pressure transducer connected to an intubated femoral artery using a PE-50 catheter. The right arm, left arm, and left leg limb leads were attached. The arterial blood pressure, heart rate, heart rhythm, and wave forms were recorded using an iWorx 214 data recorder (IX-214; iWorx Systems, Inc, Dover, NH).

\section{Heart Microcirculation Determination}

A full-field laser perfusion imager (MoorFLPI, Moor Instruments, Ltd, Devon, UK) was used to continuously quantify the microcirculatory blood flow intensity. ${ }^{21}$ The imager uses laser speckle contrast imaging, which exploits the random speckle pattern generated when tissue is illuminated by laser light. The random speckle pattern changes when blood cells move within the region of interest. When a high level of movement (fast flow) is present, the changing pattern will become more blurred, and the contrast in that region will be accordingly reduced. The contrast image is processed to produce a 16-bit color-coded image that correlates with the blood flow in 
the heart, such that blue is defined as low flow and red as high flow. The microcirculatory blood flow intensity of each region of interest was recorded as flux with the perfusion unit, which is related to the product of average speed and concentration of moving red blood cells in the heart sample volume. The negative control value was set at 0 perfusion unit (blue) and the positive value at 1000 perfusion units (red). The perfusion units were analyzed in real time using MoorFLPI software, version 3.0 (Moor Instruments, Ltd, Devon, UK).

\section{Induction of Myocardial Infarction}

Myocardial infarction was induced by coronary artery ligation for 2 hours. Midline sternotomy was performed, followed by pericardiotomy. The left anterior descending artery, close to its origin and approximately $3 \mathrm{~mm}$ away from the left coronary ostium, was ligated with 7-0 Prolene (Ethicon, Inc, Somerville, NJ), and a slipknot was tied to establish reversible coronary artery occlusion, as described previously. ${ }^{2}$

\section{Infarct Size Calculation}

After myocardial injury, $5 \mathrm{~mL}$ of methyl blue was injected through the jugular vein catheter, and the heart was harvested 2 minutes later. The heart was sliced from the base to the apex at 3-mm intervals. The right ventricle was removed from each slice, and the remaining left ventricle in each slice was weighed. The slices were incubated for 20 minutes at $37^{\circ} \mathrm{C}$ in $1 \%$ triphenyltetrazolium chloride (Sigma Aldrich, St Louis, Mo) to distinguish the infarct (pale) from the viable (red) myocardial area. ${ }^{2}$ The heart slices were photographed with a digital camera (Nikon, Tokyo, Japan). Photographic images of the heart slices were projected at fivefold magnification. The area of infarct and vital tissue for each slice were calculated using planimetric quantification and Photoshop software, version 8.0 (Adobe Systems, San Jose, Calif), in a blind fashion and corrected for the weight of the tissue slice. The fractional percentage area was then multiplied by the weight of each slice, and the results were summed to obtain the mass of the area of risk. The infarct size was expressed as a percentage of the mass of the necrotic area (pale) in the summated mass of the area of risk.

\section{In Vivo, Real-Time Chemiluminescence Recording of Heart Superoxide Anion Activity}

We measured the heart ROS in response to cardiac IR in vivo using an intravenous infusion of a superoxide anion probe, 2-methyl-6-(4methoxyphenyl)-3,7-dihydroimidazo-[1,2-a]-pyrazin-3-1-hydrochloride (MCLA) $(0.2 \mathrm{mg} / \mathrm{mL} / \mathrm{h}$, TCI-Ace, Tokyo Kasei Kogyo Co, Ltd, Tokyo, Japan) and detected using a chemiluminescence analyzing system (CLD110, Tohoku Electronic Co, Inc, Sendai, Japan $)^{1}$ and was partly modified (Figure 2, A). In brief, after surgery, the rat was maintained with a respirator and a circulating water pad at $37^{\circ} \mathrm{C}$ during photon detection. To exclude photon emission from sources other than the heart, the anesthetized rat was housed in a dark box with a shielded plate. Only the heart was left unshielded and was positioned under a reflector, which reflected the photons from the exposed heart surface onto the detector area. The MCLAenhanced chemiluminescent signal from the heart surface was recorded

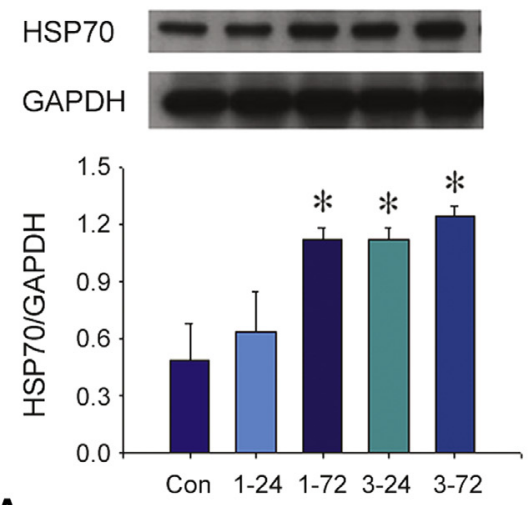

A

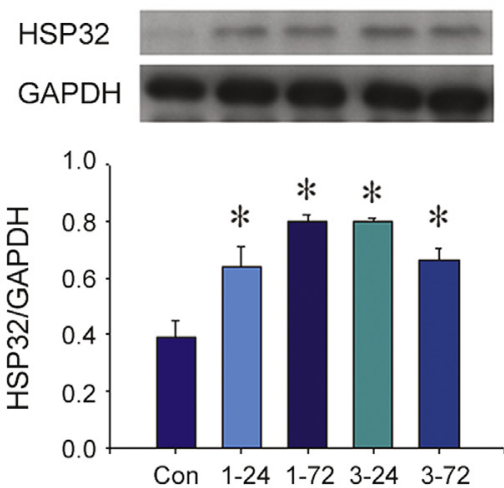

B

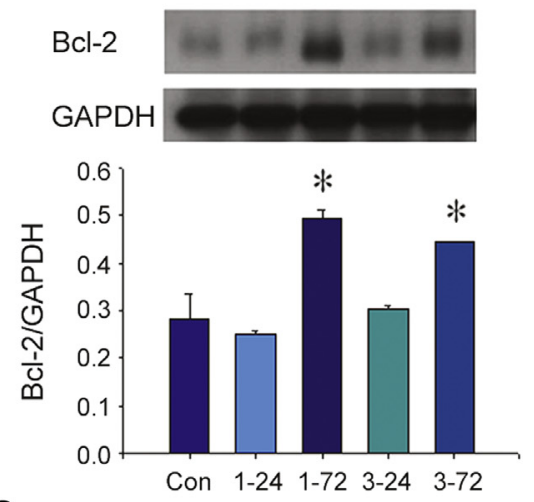

C

FIGURE 1. The responses of cardiac (A) heat shock protein (HSP)70, (B) HSP32, (C) Bcl-2, (D) Bcl-xL, and (E) MnSOD expression in the control rats (Con), after single-cycle progressive thermal preconditioning (PTP) for 24 hours (1-24) and 72 hours (1-72) or after 3 consecutive cycles of PTP 24 hours (3-24) and 72 hours (3-72). * $P<.05$ compared with the Con group. MnSOD, Manganese superoxide dismutase. 

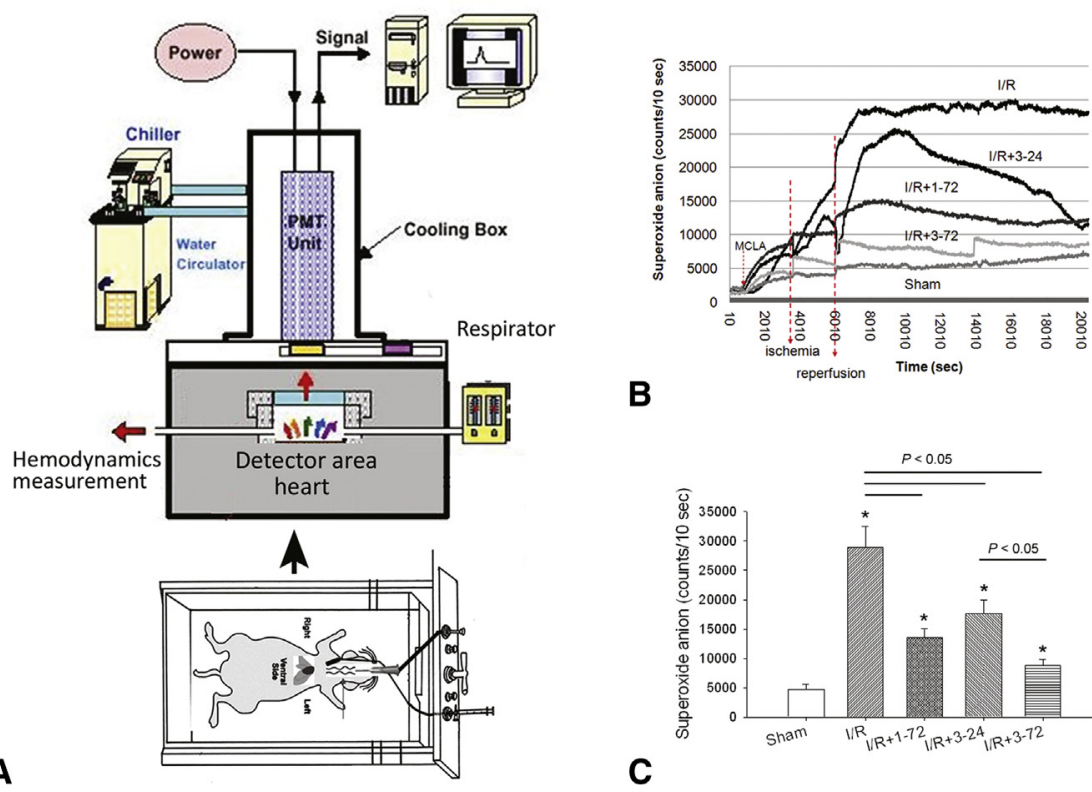

B

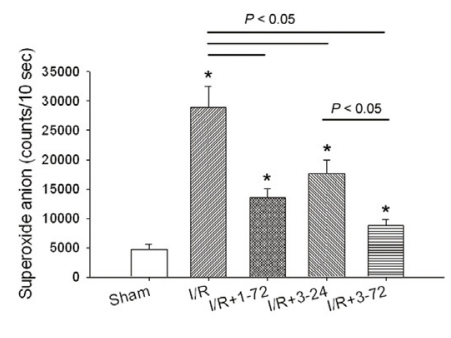

FIGURE 2. A, Setup for heart reactive oxygen species (ROS) measurement. The exposed heart of the artificially ventilated rats was placed under the detector area for measurement of cardiac ROS. The (B) original data and (C) statistical data of the ROS from the heart in the sham-control group (Sham), cardiac ischemia/reperfusion group $(I / R)$, cardiac ischemia/reperfusion after single-cycle progressive thermal preconditioning $(P T P)$ for 72 hours $(I / R+1-72)$, cardiac ischemia/reperfusion after 3 consecutive cycles of PTP for 24 hours $(I / R+3-24)$, and cardiac ischemia/reperfusion after 3 consecutive cycles of PTP for 72 hours $(I / R+3-72)$ are shown. $* P<.05$ compared with the sham-control group.

continuously using the chemiluminescence analyzer. The total ROS value was measured by the area under curve from the heart. The chemiluminescent signal obtained from $0.2 \mathrm{~mL}$ saline in $1 \mathrm{~mL}$ of MCLA $(0.2 \mathrm{mg} / \mathrm{mL})$ or $0.2 \mathrm{~mL}$ xanthine $(0.75 \mathrm{mg} / \mathrm{kg}$ body weight $) /$ xanthine oxidase $(24.8 \mathrm{mU} / \mathrm{kg}$ body weight $)$ in $1 \mathrm{~mL}$ of MCLA $(0.2 \mathrm{mg} / \mathrm{mL})$ was regarded as a negative or positive control, respectively. ${ }^{22}$

\section{Western Blotting}

We examined the protein expression in the heart tissue samples from the margin of the infarct (peri-infarct) areas using Western blotting. ${ }^{2}$

\section{In Situ Demonstration of Oxidative Stress, Autophagy, and Apoptosis Formation}

We compared the degree of apoptosis using terminal deoxynucleotidyl transferase-mediated dUTP-biotin nick end labeling (TUNEL) staining, ${ }^{1}$ LC3-II-mediated autophagy, ED-1 infiltration, and 4-hydroxynonenal (4-HNE) accumulation in the paraffin-embedded sections. ${ }^{4}$ All the histologic sections were analyzed using a Sonix Image Setup (Sonix Technology Co, Ltd, Chupei City, Hsinchu, Taiwan), including image analyzing software Carl Zeiss AxioVision, release 4.8.2 (Future Optics \& Tech. Co, Ltd, Hangzhou, China).

\section{Statistical Analysis}

All data are expressed as the mean \pm standard error of the mean. Differences within the groups were evaluated using a paired $t$ test. One-way analysis of variance was used to compare the differences among the groups. Intergroup comparisons were made using Duncan's multiple range test. The extent of myocardial infarction was corrected for the area at risk and is expressed as a percentage. A significant difference in infarct size and cardiac ROS amount was obtained from 6 rats in each group. We used SigmaPlot, version 12.0 (Systat, Chicago, Ill), for graph preparation. All statistical analyses were performed using the Statistical Package for Social Sciences software system (SPSS Inc, Chicago, Ill).

\section{RESULTS \\ PTP Enhanced HSP and Antioxidant and Antiapoptosis Proteins in the Heart}

Compared with the non-PTP control group, PTP treatment significantly enhanced cardiac HSP70 expression in the 1-72, 3-24, and 3-72 groups (Figure 1, $A$ ) and upregulated HSP32 in the 1-24, 1-72, 3-24, and 3-72 groups (Figure 1, B). Cardiac Bcl-2 and Bcl-xL were significantly upregulated only in the 1-72 and 3-72 groups (Figure 1,C). Cardiac MnSOD was significantly upregulated in the 1-72, 3-24, and 3-72 groups (Figure 1, D). According to these results, we selected the 1-72, 3-24, and 3-72 groups to challenge cardiac IR in the subsequent studies.

\section{PTP Attenuates IR-Enhanced Cardiac ROS Production}

We created an in vivo measurement of cardiac ROS associated with arterial blood pressure and heart rate from the rat (Figure 2, A). A typical graph of cardiac ROS is shown in Figure 2, $B$. The rat heart from 5 groups displayed a basal level of $\mathrm{O}_{2}^{-}$-ROS around 4000 to 8000 counts in 10 seconds during MCLA infusion. After IR, the $\mathrm{O}_{2}{ }^{-}$-ROS level was enhanced to 30,000 counts in 10 seconds in the non-PTP group, 20,000 to 25,000 counts in 10 seconds in the 3-24 group, 15,000 counts in 10 seconds in the 1-72 group, and 10,000 counts in 10 seconds in the 3-72 group. The increased $\mathrm{O}_{2}{ }^{-}$-ROS level was greater in the non-PTP group than in the 


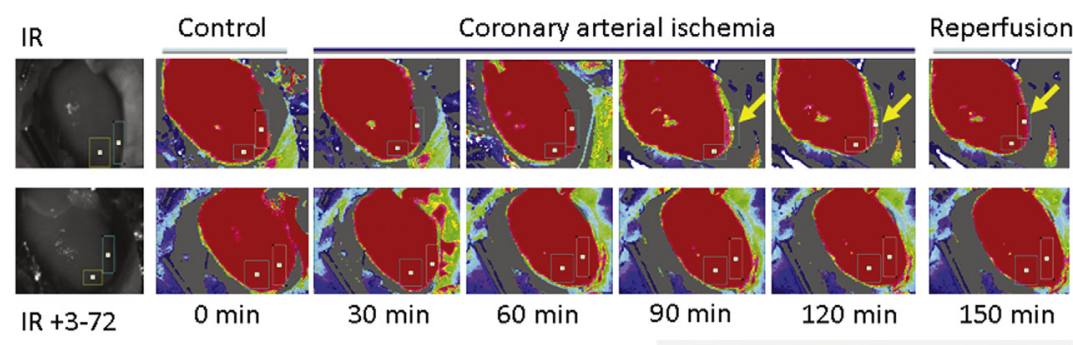

A

IR

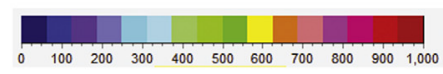

$\mathrm{IR}+3-72$

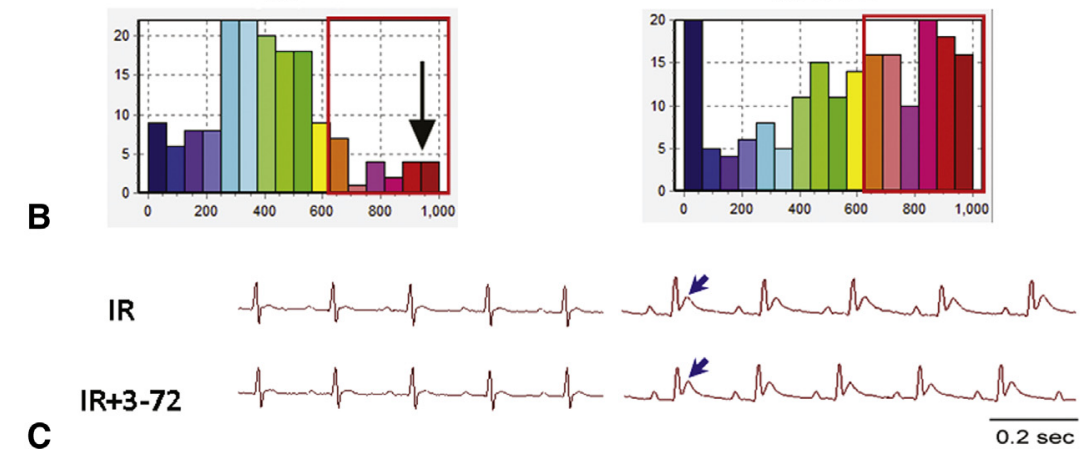

IR

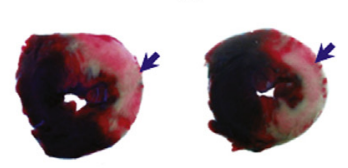

$\mathrm{IR}+3-72$
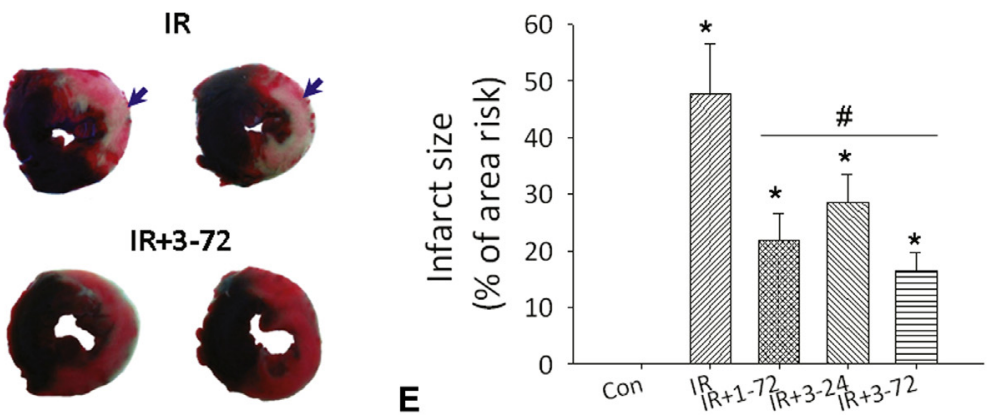

FIGURE 3. Progressive thermal preconditioning (PTP) effect on ischemia/reperfusion (IR)-induced alteration in (A), (B) cardiac microcirculation, (C) electrocardiographic findings, and (D) (E) infarct size. Marked differences were seen in the cardiac microcirculation and infarct size between the IR group and the IR with PTP treatment group. C, IR induced marked ST-segment elevation in the electrocardiographic wave in the IR and IR plus 3 consecutive cycles of PTP for 72 hours (+3-72) groups. E, PTP significantly decreased the infarct size compared with that in the IR group. * $P<.05$, compared with the control group; $\# P<.05$, compared with the IR group. Con, Control rats; $I / R+3-24$, ischemia/reperfusion after 3 consecutive cycles of PTP for 24 hours.

3-24 group, which was greater than that in the 1-72 group, which was greater than that in the 3-72 group (Figure 2, C).

\section{PTP Improved IR-Depressed Microcirculation and IR-Increased Infarct Size}

During the control, ischemia, and reperfusion periods, the sham-control heart displayed a consistent red color throughout the experiment (data not shown). In the nonPTP control rat, IR markedly decreased the blood perfusion to a pink or green in the lower region of the left ventricle (Figure 3, A). In the 3-72 group, PTP restored the microcirculation to red in the left lower region of the ventricle in response to IR. The quantified data displayed a greater ventricular blood perfusion unit in the 3-72 group than in the non-PTP control group (Figure 3, $B$ ).
The baseline electrocardiographic (ECG) wave was similar in the non-PTP control and 3-72 groups (Figure 3, $C)$. IR induced a similar ST-segment elevation in the ECG wave in the non-PTP and 3-72 groups. IR increased the infarct size in the non-PTP control and 3-72 groups (Figure $3, D$ ). However, the infarct area was significantly depressed in the 3-72 group compared with the non-PTP control group. The infarct size was greater in the non-PTP than in the 3-24 group, which was greater than in the 1-72 group, which was greater than in the 3-72 group (Figure 3, E).

\section{PTP Reduced IR-Enhanced Oxidative Injury}

IR significantly enhanced the cardiac $\mathrm{Bax} / \mathrm{Bcl}-2$ ratio, cleaved caspase 3, poly-(ADP-ribose)-polymerase, LC3-II, 

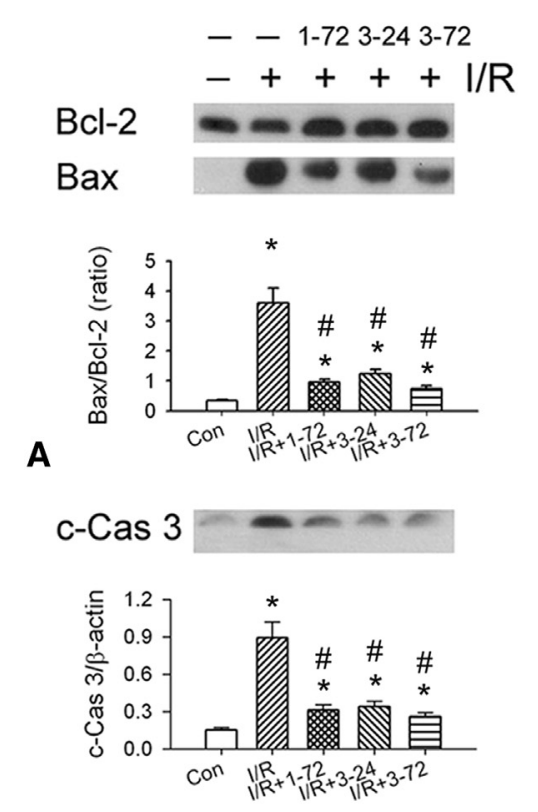

B

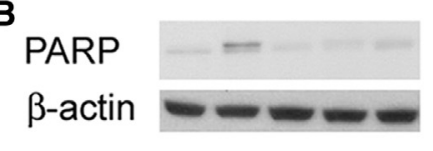

C

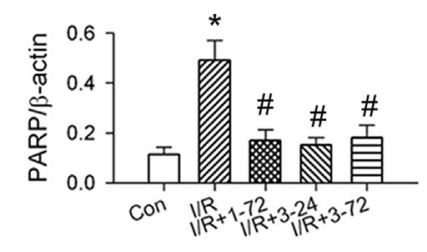

$$
--1-723-243-72
$$$$
-++++1 / R
$$

LC3-II

$\beta$-actin
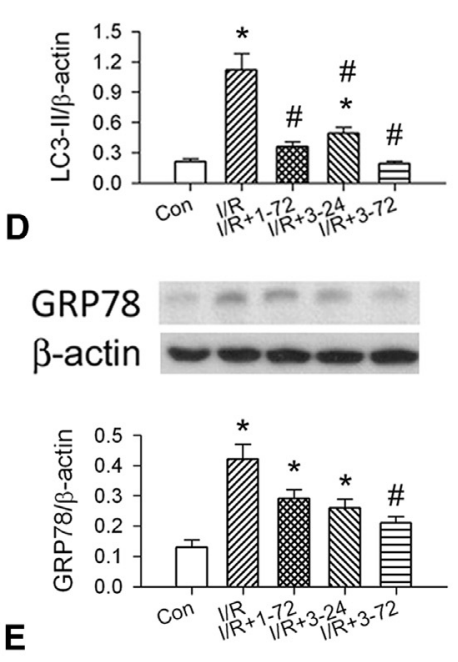

\section{$\mathrm{E}$}

FIGURE 4. Progressive thermal preconditioning (PTP) effect on (A-C) apoptosis-related proteins, (D) autophagy-LC3-II, (E) endoplasmic reticulum stress glucose-regulated protein $(G R P) 78$, (F) mitochondrial Bcl-2 $(m-B c l-2),(\mathrm{G})$ cytosolic cytochrome $\mathrm{C}(\mathrm{c}-\mathrm{Cyt} \mathrm{C})$, and $(\mathrm{H})$ nicotinamide adenine dinucleotide phosphate oxidase gp91 subunit ( $g p 91)$ expression levels. $* P<.05$ versus control (Con) group; $\# P<.05$ versus ischemia/reperfusion $(I / R)$ groups. 1 72, 24 Hours after single-cycle PTP in a $42^{\circ} \mathrm{C}$ water bath; 3-24, 24 hours after 3 consecutive cycles of PTP in a $42^{\circ} \mathrm{C}$ water bath; 3-72, 72 hours after 3 consecutive cycles of PTP in a $42^{\circ} \mathrm{C}$ water bath; $H s p$, heat shock protein; $c$-Cas 3, caspase 3; PARP, poly-(ADP-ribose)-polymerase.

ER stress-78-kDa glucose-regulated protein 78, cytosolic cytochrome $\mathrm{C}$, and NADPH oxidase gp91 expression and decreased mitochondrial Bcl-2 expression (Figure 4) and mitochondrial Bcl-2 expression and depressed IR-enhanced $\mathrm{Bax} / \mathrm{Bcl}-2$ ratio, cleaved caspase 3, poly-(ADP-ribose)-polymerase, LC3-II, 78-kDa glucose-regulated protein 78, cytosolic cytochrome $\mathrm{C}$, and gp91 expression.

\section{PTP Reduced IR-Enhanced Cardiac Inflammation, Oxidative Stress, Autophagy, and Apoptosis}

IR significantly enhanced cardiac ED-1 infiltration, 4-HNE, LC3-II, and TUNEL stains in the non-PTP control and PTP groups (Figure 5). However, the 3-72 PTP treatment group had significantly decreased IR-enhanced cardiac ED-1, 4-HNE, LC3-II, and TUNEL stains compared with the non-PTP control group (Figure 5).

\section{DISCUSSION}

According to our previous data, ${ }^{16,17}$ a whole body hyperthermia challenge (immediate immersion in a $42^{\circ} \mathrm{C}$ water bath for 15 minutes) significantly induces temporary hypertension, tachycardia, ER stress, and oxidative stress. However, PTP treatment significantly attenuated the enhancement of hypertension, tachycardia, $\mathrm{ER}$, and oxidative stress during hot water immersion. Our data showed that PTP confers cardioprotection through the combination of antioxidant, antiapoptotic, and antiautophagic signaling in a frequency-dependent manner. These data have implicated its safety and efficacy in response to hot water challenges. The application of PTP efficiently provided cardiovascular protection against $\mathrm{FeCl}_{3}$-induced arterial thrombosis ${ }^{16,17}$ and cardiac IR injury in the present study. 

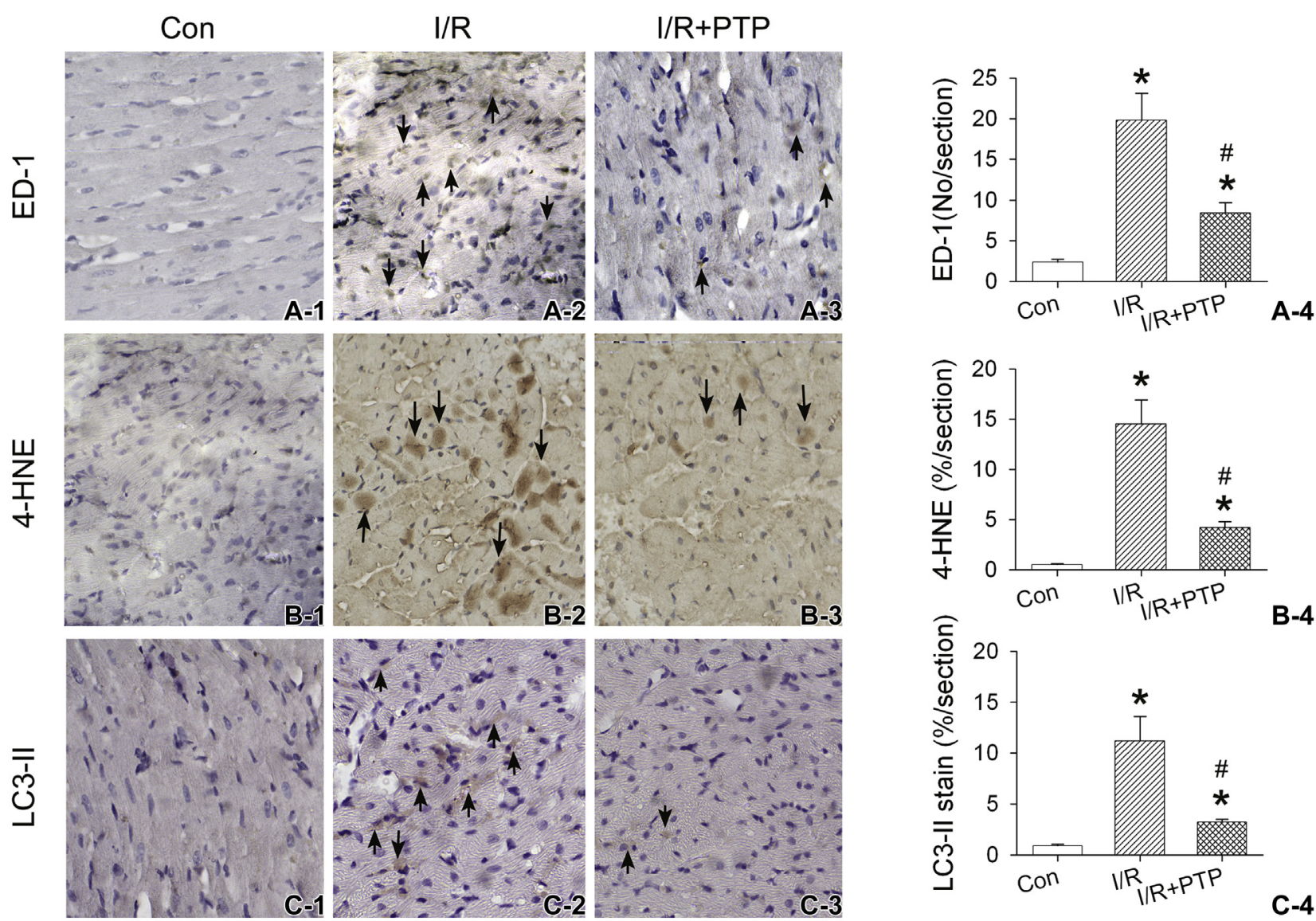

B-4
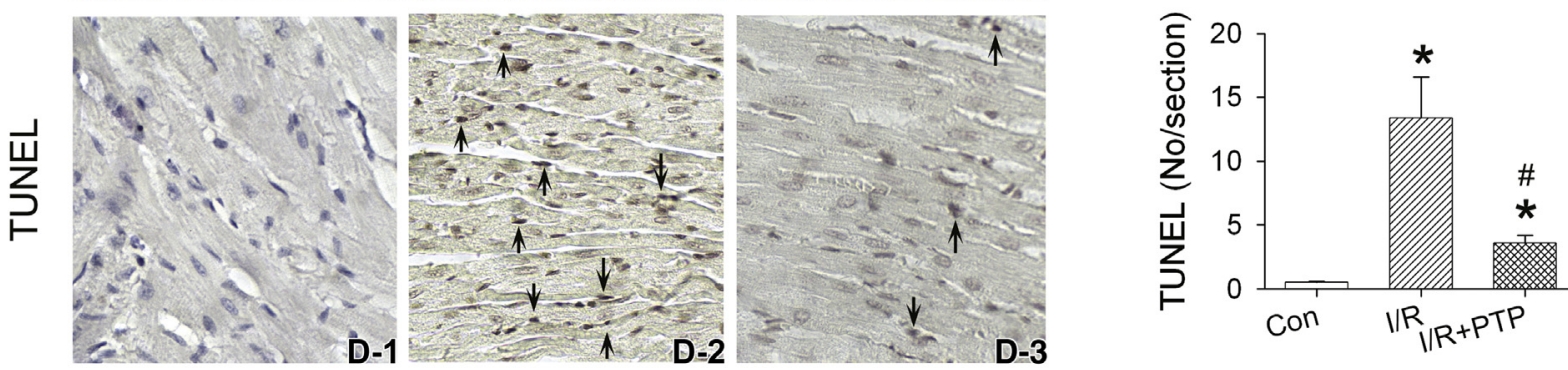

C-4

FIGURE 5. Progressive thermal preconditioning $(P T P)$ effect on inflammation, oxidative stress, autophagy, and apoptosis in the ischemia/reperfusion $(I / R)$ heart. A, ED-1 expression; B, 4-hydroxynonenal (4-HNE) expression; C, LC3-II expression; D, apoptosis expression determined by terminal deoxynucleotidyl transferase-mediated dUTP-biotin nick end labeling (TUNEL) stain. ${ }^{*} P<.05$ versus control (Con) group; \#P<.05 versus I/R group.

The present study has demonstrated that PTP enhanced the antioxidant, antiapoptotic, and antiautophagic actions in a frequency-dependent manner. The 3-72 group displayed the strongest cardiac protection, greater than that in the 3-24, 1-24, and 1-72 groups. These beneficial effects reduced cardiac ROS, ED-1 infiltration, 4-HNE, apoptosis, and autophagy in the IR heart, leading to a decrease in the infarct size. PTP also exerted a beneficial effect in restoring cardiac microcirculation in response to IR. Diverse stress preconditioning stimuli enhanced HSP70 and endothelial nitric oxide (NO) synthase expression through the phosphatidylinositol 3-kinase/Akt signaling pathway to increase NO production and vasodilation. ${ }^{17,18}$ Whether PTP restores cardiac microcirculation by way of the phosphatidylinositol 3-kinase/Akt/endothelial NO synthase signaling and the increased $\mathrm{NO}$ level requires additional studies to be determined. In addition, the importantly critical factor for the induction and enforcement of the PTPinduced several defense mechanisms will be explored in the future.

In the present study, we also found that cardiac IR induced a similar symptom of abnormal ECG findings of ST-segment elevation in all the rats. However, the cardiac microcirculation, infarct size, and molecular mechanisms and pathologic findings displayed a differential pattern. These findings suggest that the ECG can implicate IR injury 
in the heart; however, the ECG findings might not actually indicate the degree of cardiac injury.

ROS play an important role in IR injury; however, obtaining direct proof has been hampered by the high reactivity and short biologic half-life of ROS. Several techniques have been used for the study of the production and mechanisms of ROS in IR tissue, including electron spin resonance spectroscopy, the nitro blue tetrazolium method, DAB- $-\mathrm{Mn}^{2+}$ cytochemistry, and measurement of the induction or depletion of endogenous ROS scavengers. ${ }^{1}$ In the present study, for the first time, we used an enhanced chemiluminescent method to study real-time ROS production directly in the heart in vivo. The differing amount of ROS correlated with the degree of injury, indicating the technique is reliable. Using an in situ 4-HNE stain, we also found that the cellular source of ROS synthesis was possibly from the IR cardiomyocytes and infiltrated ED-1. Enhanced NADPH oxidase gp91 expression and cytosolic cytochrome $\mathrm{C}$ release in cardiomyocytes both contribute to the exacerbated ROS production, leading to subsequent inflammation and programmed cell death. The use of PTP can attenuate NADPH oxidase gp91 expression and cytosolic cytochrome $\mathrm{C}$ release and result in the inhibition of ROS production.

The generation of ROS on reperfusion has been cited as one of the major causes of IR injury to induce oxidative stress, ER stress, mitochondrial dysfunction, leukocytemediated inflammation, and autophagy and apoptosis. ${ }^{3,4}$ Myocardial protection can be induced by ischemic preconditioning and delta-opioid receptor activation in the isolated working rat heart for improving the postischemic recovery of aortic flow. ${ }^{23}$ Pharmacologic preconditioning using darbepoetin-alfa achieved cardioprotection through the upregulation of both $\mathrm{Bcl}-\mathrm{xL}$ and $\mathrm{Bcl}-2$ to decrease ROS production, lower cleaved caspase-3, and increase phosphorylated-Bad. ${ }^{24}$ Pharmacologic preconditioning using hydrogen sulfate resulted in cardioprotection by way of increased expression of antioxidants (heme oxygenase1 and thioredoxin 1), HSP90, HSP70, Bcl-2, Bcl-xL, and cyclooxygenase- 2 and inactivated the proapoptogen Bad. ${ }^{25}$ These defense mechanisms decreased infarct size, circulating troponin I levels, and oxidative stress. ${ }^{25}$ Latephase ischemic preconditioning protects the heart against IR injury by upregulating MnSOD protein expression, preserving mitochondrial oxygen metabolism, and attenuating IR injury. ${ }^{8}$ Selectively overexpressed MnSOD in the heart mitochondria of the transgenic mice reduced the infarct size in vivo in a left coronary artery ligation model. ${ }^{26}$ In the present study, PTP conferred cardioprotection by improving cardiac microcirculation and upregulating HSP32, HSP70, Bcl-2, Bcl-xL, and MnSOD and preserving mitochondrial function and integrity. These defense mechanisms triggered antioxidant, anti-inflammation, anti-ER stress, antiapoptosis, and antiautophagy signaling by PTP and, consequently, protected the heart against IR injury.
Mitochondria and NADPH oxidase are the target and source of ROS, which play an important role in physiologic signaling mechanisms and in the regulation of apoptotic and autophagy pathways. ${ }^{4}$ Increased ROS and Bax or decreased Bcl-2 and Bcl-xL open the mitochondria permeability transition pore through the outer mitochondrial voltage-dependent anion conductance channel to release cytochrome $\mathrm{C}$ into cytosol., ${ }^{2,27-29}$ Increased mitochondrial $\mathrm{Bax}$ and $\mathrm{O}_{2}{ }^{-}$and decreased mitochondrial $\mathrm{Bcl}-2$ or Bcl-xL trigger cytosolic cytochrome $\mathrm{C}$ release, possibly through mitochondria permeability transition pore opening to induce apoptosis and autophagy in the IR tissue. ${ }^{2,3,27}$ PTP enhanced several defense mechanisms (upregulating MnSOD, Bcl-2, and Bcl-xL) to counteract IR-induced mitochondrial dysfunction by preserving mitochondrial $\mathrm{Bcl}-2$ expression and decreasing cytosolic cytochrome $\mathrm{C}$ release, suggesting its role in mitochondrial protection.

How does superficial heating of the skin affect inner organs such as the heart? Incremental changes in the temperature $\left(28^{\circ}-42.5^{\circ} \mathrm{C}\right)$ of the anterior left ventricular wall in a canine affected the myocardial pressure and length areas but did not change the coronary blood flow or distribution. ${ }^{30}$ In traditional Chinese medicine, heat stimulation of certain skin areas (Hsuch-Tao) has been used in the treatment of various functional disorders of the inner organs. The mechanism of action is still unknown. It has been suggested that somatic sensory stimuli (warm receptor activation by thermal stimulation) are capable of influencing neural mechanisms in the central nervous system and, in turn, through neural and/or humoral messages to affect the visceral effectors of the body. For example, thermal stimulation of a different area of the skin could alter the renal sympathetic efferent activity to the kidney and increased the urinary kallikrein excretion. It requires additional studies to explore the PTP-induced effects. For a possible application to clinical trials, we could adapt mild hyperthermia methods such as PTP for triggering hyperthermia-induced protection in the patients before surgery. For the prevention of exacerbated hemodynamic variation during whole body hyperthemia, a safe strategy for progressive water bath immersion at $\leq 42^{\circ} \mathrm{C}$ can be recommended. However, the cardiovascular protection might be attenuated because of the short duration and low temperature $\left(<42^{\circ} \mathrm{C}\right)$. Considering the safety and efficacy, our PTP with 3 steps of $42^{\circ} \mathrm{C}$ bathing attenuated the adverse effects, such as tachycardia or blood pressure fluctuations, occurring in whole body hyperthermia but still conferred cardiovascular protection. The increased PTP frequency (3 times daily in the present study) seemed to enforce additional cardiovascular potential. PTP could be used as prophylaxis to reduce or prevent cardiovascular injury. However, additional studies are required to explore theses issues for clinical application in the future. 


\section{CONCLUSIONS}

Modified PTP provided an effective, safe, and available strategy to protect against cardiac IR injury by the enforced antioxidant, anti-inflammatory, antiapoptotic, antiautophagic, and anti-ER stress mechanisms.

\section{References}

1. Chien CT, Lee PH, Chen CF, Ma MC, Lai MK, Hsu SM, et al. De novo demonstration and co-localization of free-radical production and apoptosis formation in rat kidney subjected to ischemia/reperfusion. JAm Soc Nephrol. 2001;12:973-82.

2. Chien CT, Chang TC, Tsai CY, Shyue SK, Lai MK. Adenovirus-mediated bcl-2 gene transfer inhibits renal ischemia/reperfusion induced tubular oxidative stress and apoptosis. Am J Transplant. 2005;5:1194-203.

3. Chien CT, Shyue SK, Lai MK. Bcl-xL augmentation potentially reduces ischemia/reperfusion induced proximal and distal tubular apoptosis and autophagy. Transplantation. 2007;84:1183-90.

4. Chung SD, Lai TY, Chien CT, Yu HJ. Activating Nrf-2 signaling depresses unilateral ureteral obstruction-evoked mitochondrial stress-related autophagy, apoptosis and pyroptosis in kidney. PLoS One. 2012;7:e47299.

5. Chen YS, Chien CT, Ma MC, Tseng YZ, Lin FY, Wang SS, et al. Protection "outside the box" (skeletal remote preconditioning) in rat model is triggered by free radical pathway. J Surg Res. 2005;126:92-101.

6. Sorrentino SA, Doerries C, Manes C, Speer T, Dessy C, Lobysheva I, et al. Nebivolol exerts beneficial effects on endothelial function, early endothelial progenitor cells, myocardial neovascularization, and left ventricular dysfunction early after myocardial infarction beyond conventional $\beta 1$-blockade. J Am Coll Cardiol. 2011;57:601-11.

7. Bao W, Behm DJ, Nerurkar SS, Ao Z, Bentley R, Mirabile RC, et al. Effects of p38 MAPK inhibitor on angiotensin II-dependent hypertension, organ damage, and superoxide anion production. J Cardiovasc Pharmacol. 2007;49:362-8.

8. Li Y, Cai M, Xu Y, Swartz HM, He G. Late phase ischemic preconditioning preserves mitochondrial oxygen metabolism and attenuates post-ischemic myocardial tissue hyperoxygenation. Life Sci. 2011;88:57-64.

9. Halliwell B. The antioxidant paradox. Lancet. 2000;355:1179-80.

10. George J, Greenberg S, Barshack I, Singh M, Pri-Chen S, Laniado S, et al. Accelerated intimal thickening in carotid arteries of balloon-injured rats after immunization against heat shock protein 70. J Am Coll Cardiol. 2001;38:1564-9.

11. McCormick PH, Chen G, Tierney S, Kelly CJ, Bouchier-Hayes DJ. Clinically relevant thermal preconditioning attenuates ischemia-reperfusion injury. J Surg Res. 2003;109:24-30.

12. Yellon DM, Pasini E, Cargnoni A, Marber MS, Latchman DS, Ferrari R. The protective role of heat stress in the ischaemic and reperfused rabbit myocardium. J Mol Cell Cardiol. 1992;24:895-907.

13. Kubota K, Tamura K, Take H, Kurabayashi H, Shirakura T. Acute myocardial infarction and cerebral infarction at Kusatsu-spa. Nippon Ronen Igakkai Zasshi. 1997;34:23-9.

14. Sobajima M, Nozawa T, Thori H, Shida T, Ohori T, Suzuki T, et al. Repeated sauna therapy improves myocardial perfusion in patients with chronically occluded coronary artery-related ischemia. Int J Cardiol. 2013;167:237-43.
15. Okada M, Hasebe N, Aizawa Y, Izawa K, Kawabe J, Kikuchi K. Therma treatment attenuates neointimal thickening with enhanced expression of heat-shock protein 72 and suppression of oxidative stress. Circulation 2004;109:1763-8.

16. Huang SC, Tsai YF, Cheng YS, Liu KH, Li PC, Chien CT. Vascular protection with less activation evoked by progressive thermal preconditioning in adrenergic receptor-mediated hypertension and tachycardia. Chin J Physiol. 2009;52: 419-25.

17. Li PC, Yang CC, Hsu SP, Chien CT. Repetitive progressive thermal preconditioning hinders thrombosis by reinforcing phosphatidylinositol 3-kinase/Akt-dependent heat-shock protein/endothelial nitric oxide synthase signaling. J Vasc Surg. 2012;56:159-70.

18. Yeh CH, Hsu SP, Yang CC, Chien CT, Wang NP. Hypoxic preconditioning reinforces HIF-alpha-dependent HSP70 signaling to reduce ischemic renal failure-induced renal tubular apoptosis and autophagy. Life Sci. 2010;86: 115-23.

19. Qian H, Yang Y, Wang X. Curcumin enhanced Adriamycin-induced human liverderived hepatoma G2 cell death through activation of mitochondria-mediated apoptosis and autophagy. Eur J Pharm Sci. 2011;43:125-31.

20. Adams JM, Cory S. The Bcl-2 protein family: arbiters of cell survival. Science. 1998;281:1322-6.

21. Yeh YC, Wang MJ, Lin CP, Fan SZ, Tsai JC, Sun WZ, et al. Enoxaparin sodium prevents intestinal microcirculatory dysfunction in endotoxemic rats. Crit Care. 2012; 16:R59.

22. Chen CF, Tsai SY, Ma MC, Wu MS. Hypoxic preconditioning enhances renal superoxide dismutase levels in rats. $J$ Physiol. 2003;552:561-9.

23. Karck M, Tanaka S, Bolling SF, Simon A, Su TP, Oeltgen PR, et al. Myocardial protection by ischemic preconditioning and delta-opioid receptor activation in the isolated working rat heart. J Thorac Cardiovasc Surg. 2001;122: 986-92.

24. Gross A, Jockel J, Wei MC, Korsmeyer SJ. Enforced dimerization of BAX results in its translocation, mitochondrial dysfunction and apoptosis. EMBO J. 1998;17: 3878-85.

25. Schlecht-Bauer D, Antier D, Machet MC, Hyvelin JM. Short- and long-term cardioprotective effect of darbepoetin-alpha: role of Bcl-2 family proteins. J Cardiovasc Pharmacol. 2009;54:223-31.

26. Calvert JW, Jha S, Gundewar S, Elrod JW, Ramachandran A, Pattillo CB, et al. Hydrogen sulfide mediates cardioprotection through Nrf2 signaling. Circ Res. 2009; 105:365-74.

27. Chen Z, Siu B, Ho YS, Vincent R, Chua CC, Hamdy RC, et al. Overexpression of MnSOD protects against myocardial ischemia/reperfusion injury in transgenic mice. J Mol Cell Cardiol. 1998;30:2281-9.

28. Antonsson B, Montessuit S, Lauper S, Eskes R, Martinou JC. Bax oligomerization is required for channel-forming activity in liposomes and to trigger cytochrome C release from mitochondria. Biochem J. 2000;345:271-8.

29. Marzo I, Brenner C, Zamzami N, Susin SA, Beutner G, Brdiczka D, et al. The permeability transition pore complex: a target for apoptosis regulation by caspases and bcl-2-related proteins. J Exp Med. 1998;187:1261-71.

30. D'Ambra MN, Magrassi P, Lowenstein E, Kyo S, Austen WG, Buckley MJ, et al Myocardial temperature variation: effect on regional function and coronary flow in dogs. Am J Physiol. 1987;252:H448-55. 